\title{
Comparative Analysis of Intel Pentium 4 and IEEE/EMC TC-9/ACEM CPU Heat Sinks
}

\author{
Junwei Lu and Xiao Duan \\ Griffith University,Brisbane, QLD 4111, Australia \\ j.lu@griffith.edu.au and xiao.duan@student.griffith.edu.au
}

\begin{abstract}
This paper presents finite element frequency domain results for electromagnetic radiation emitted from high power microelectronic circuits connected to a heat sink. The heat sink model associated with one of the IEEE EMC TC-9 challenging problems, 2000-4 CPU heat sink, has been used to investigate different grounding configurations. A new simulation model for the Intel P4 CPU heat sink is proposed. In contrast to the 2000-4 EMC challenging model, Intel P4 CPU heat sink model exhibited different results. A resonant frequency of $2.6 \mathrm{GHz}$ with a reflection coefficient of $\mathbf{- 8 . 3 \mathrm { dB }}$ was found for the Intel P4 CPU heat sink, which is close to the operating frequency of $2.4 \mathrm{GHz}$ for IEEE and Bluetooth wireless communication systems. The comparison of frequency sweeping results shows that the reflection coefficient was found to be $-16 \mathrm{~dB}$ at $3.6 \mathrm{GHz}$ and $-20 \mathrm{~dB}$ at 3.4GHz from conventional CPU heat sink. The CPU heat sink can perform as an efficient radiator at these frequencies.
\end{abstract}

\section{INTRODUCTION}

Modern silicon wafer fabrication facilities easily produce component densities that exceed 1 million transistors per die. Some components consume a significant amount of DC power. Computer components such as the Intel and AMD processors require a separate cooling system provided by a fan built into their heat sink or by a fan or cooling devices located adjacent to the processor. Since these high-power and high-speed processors are being implemented in more designs, special design techniques are now required for EMI suppression and heat removal at the component level. As a result, traditional techniques are no longer valid for representing electrical behaviour and full-wave numerical analysis tools are required. Obviously, full wave numerical analysis tools are required to model the radiated emissions given that traditional models are unable to do so.

In this paper we consider RF domain problems that take into account the CPU heat sink, and the thermally conducting compound at the interface between the component and heat sink. A conventional heat sink model with grounded metal heat sink (EMC challenging model, standard problem 2000-4) and a new model with insulated metal heat sink (Intel Pentium 4) are analyzed in the RF domain. A frequency domain approach technique is used in the simulation.

\section{Full WaVE EM MOdeling TECHNIQUES}

To obtain a full solution to Maxwell's equations for a structure of arbitrary size, a full-wave technique is required. A full-wave computational technique provides a complete solution to Maxwell's equations within the computational space for all conductors and materials. Full wave techniques are more complex than quasi-static techniques, but they are also generic in nature and have fewer limitations in their use.

Each of the various full-wave techniques, has its own strengths, and weaknesses. The limitations of practical fullwave techniques vary from technique to technique and on the level of detail required in the constructed model. Each fullwave modeling technique is limited to particular types of models. Currently there is no single numerical technique that will solve all modeling problems that an EMC engineer is likely to encounter. The type of numerical technique chosen depends on the application problem.

The solution to a CPU heat sink problem can be found in either the frequency or time domain. Solutions obtained in the time domain use a Fourier transform to provide output data as a function of frequency [1]. Since frequency domain codes must be run for each frequency of interest, sometimes a frequency sweeping technique is required to obtain the solution in a certain range of frequencies [2, 3]. Two different wave equations are normally used in EMC computer modeling to solve EM radiation problems in open space.

\section{A. Time-Domain EM Modeling Techniques}

Time-domain techniques use a band-limited impulse to generate a wide frequency range excitation source as an input to the simulation. The result obtained from a time-domain code is the model's response to this impulse. A Fourier transform is applied to the time-domain data when frequencydomain information is required. While there is a large number of driving wave forms available, the most common waveform is in the form of a Gaussian pulse. A simple Gaussian pulse contains energy from direct current $(\mathrm{dc})$ to a defined upper frequency. Another form is the differentiated Gaussian pulse that contains no dc component and has a 6-dB/oct falling decay value with decreasing frequency and the normal fast roll-off at high frequencies [2]. The time domain vector wave equation for $\boldsymbol{E}$ field can be obtained from Maxwell's equations, that is:

$$
\nabla \times \frac{1}{\mu} \nabla \times E+\sigma_{e} \frac{\partial E}{\partial t}+\varepsilon \frac{\partial^{2} E}{\partial t^{2}}=-\frac{\partial J}{\partial t}
$$

where $\boldsymbol{J}$ is source current, $\sigma_{e}$ is the effective conductivity, $\mu$ and $\varepsilon$ are the permeability and permittivity of the problem space respectively. 


\section{B. Frequency-Domain EM Modeling Techniques}

Frequency-domain codes solve for one frequency at a time. This is usually adequate for antenna work and for examining specific issues. Frequency-domain codes are in general faster than their time-domain cousins. Therefore, several frequencydomain simulations can usually be run in the time it would take for a single time-domain simulation. A further benefit to using frequency-domain codes is their capacity to use larger meshes for the lower frequencies, which in turn permits a shorter computation time. To cover a wide frequency range with frequency-domain codes, a number of simulations are required. One major limitation of frequency domain method is the frequency range over which the model is valid. This limitation is primarily imposed by how finely the problem is partitioned and how close the elements are to the edges of the computational space. Computation error can be introduced if excitation sources extend beyond the valid frequency range. In addition, the frequency sweeping technique may cause other error during the frequency domain simulation, as the mash size and the space between radiation source and radiation boundary may not satisfy all interested frequencies. It should be noted that there are interpolation techniques available that minimize the number of simulations required. However, these interpolation techniques must be used with care to ensure a resonance effect is not omitted. The frequency domain vector wave equation for $\boldsymbol{E}$ field can be derived as:

$$
\nabla \times \frac{1}{\mu} \nabla \times E+\omega^{2} \varepsilon E=-j \omega J
$$

where $\omega$ is angular frequency, $\boldsymbol{J}$ is source current, $\sigma_{\mathrm{e}}$ is the effective conductivity, $\mu$ and $\varepsilon$ are the permeability and permittivity of the problem space respectively.

\section{EMC COMPUTER MOdeling OF CPU HeAt SinK}

The power generated from processor currents can reach levels of $50 \mathrm{~W}$. Also, clocking speeds are over $1 \mathrm{GHz}$. This combination of switching frequency and power level in conjunction with the layout of the common mode current paths through the heat sinks results in significant radiated EMI. As a result, EMC engineers need to understand the cause of radiated emissions from heat sinks, and find ways to reduce them whenever possible.

A joint IEEE/EMC Society Technical Committee (TC-9) and Applied Computational Electromagnetic Society (ACEM) have partnered in an effort to develop specific standard problems [4]. The CPU heat sink problem is one of the EMC challenging problems. The purpose of these problems is to provide EMI/EMC engineers and vendors with a way of determining the validity and accuracy of their EM modeling software. To model the heat sink structure, it is useful to break down the structure into the following three regions; the ground plane, the source region and the heat sink. A realistic representation of a VLSI device must take into account the electromagnetic source characteristics and an actual physical model e.g. a conducting patch [1]. Real heat sinks have fins to increase the thermal conductance. Brench [5] found that the heat sink could be modeled as a solid block. One approach from Das and Roy [6] models the source as a monopole that cuts through a VLSI device. Das and Roy tested three cases: ground plane monopole, ground plane monopole through a VLSI and element through a VLSI with a heat sink. From the experimental results, Das and Roy concluded that as a first approximation, a monopole could be used to model the characteristics of a heat sink structure.

The Intel Pentium 4 Processor is placed in a 478-Pin Package as shown in Fig. 1 and can operate at 1.40, 1.50, $1.60,1.70,1.80,1.90$ and $2 \mathrm{GHz}$ [7]. In the Intel P4 configuration, a heat spreader is located on top of the VLSI and the heat spreader is isolated from the VLSI packaging. Therefore, a different numerical model needs to be employed, and its simulation model consisting of a multi layered structure for the P4 and heat sink system is developed using patch antenna driven concept as shown in Fig. 2 [8-9].

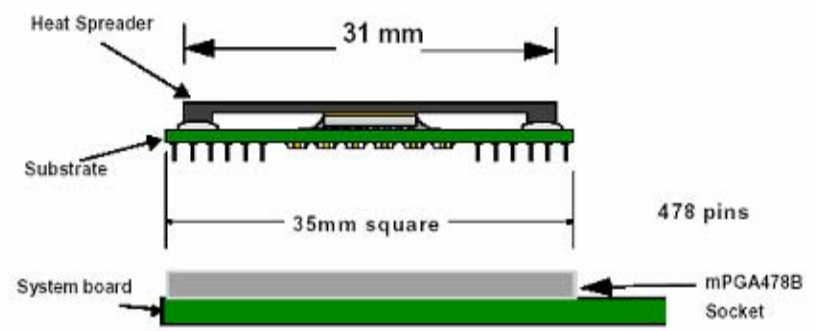

Fig. 1 Configuration of Intel P4 CPU [7].

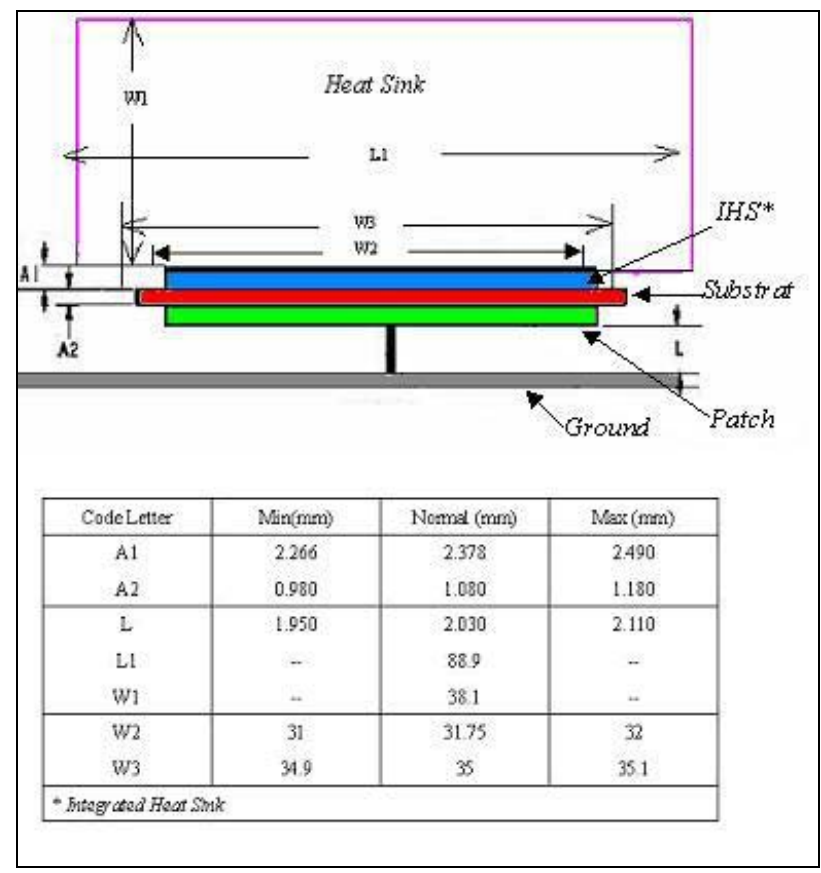

Fig. 2 Computation model of Intel P4 CPU and heat sink, where the following materials are used; IHS: copper, Substrate: polyamide, Patch: copper, Heat sink: aluminum, Ground plane: copper. 
Obviously, the Intel P4 processor has a different packaging and structural configuration compared with conventional CPU configurations and the IEEE challenge model e.g. 2000-4, as illustrated in Fig. 3 [4].

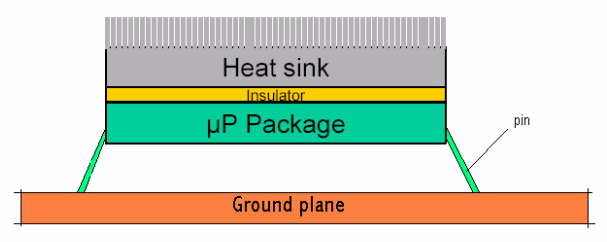

(a) Configuration of conventional CPU and heat sink

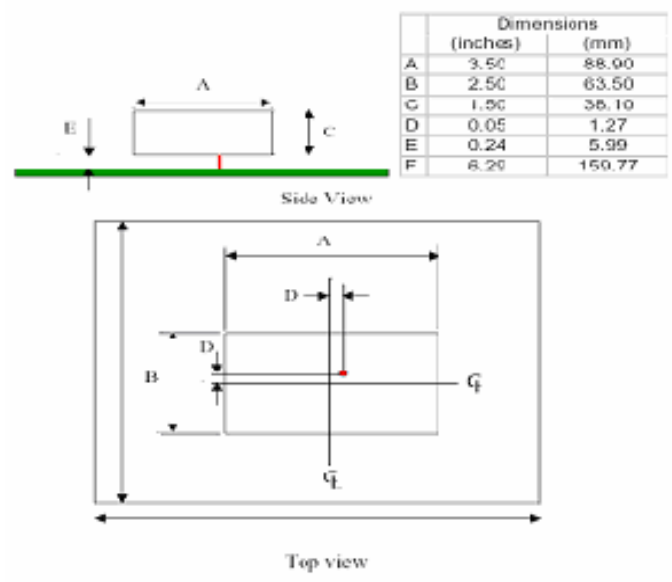

(b) Computer simulation model of CPU and heat sink

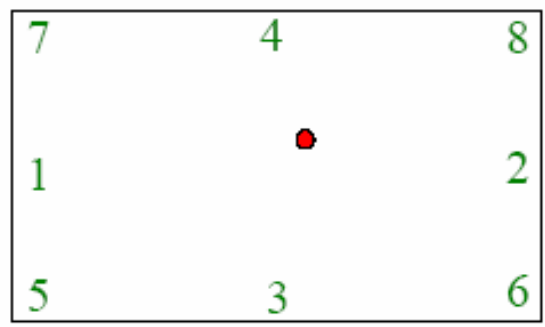

(c) Grounding position

Fig. 3. CPU heat sink configuration of EMC TC-9 Challenge Problem [4].

\section{COMPutation Results of CPu Heat SinK}

\section{A. Analysis of Intel P4 CPU Heat Sink}

The computation model of the Intel P4 CPU heat sink shown in Fig. 2 was simulated in the frequency domain using a commercial EM simulator; High Frequency Structure Simulator (HFSS) [10], which employs a finite element frequency domain method. In this computation model, a radiation boundary is used to simulate an open boundary problem that allows waves to radiate infinitely into space. A radiation surface does not have to be spherical, but it must be (a) exposed to the background, (b) convex with regard to the radiation source, and (c) located at least a quarter wavelength from the radiating source. The shape of the boundary in our simulation model is spherical. The space in the sphere is the computational domain which is related to the frequency range we are interested in. The exciting port we used in our simulation is a Lumped port [10], where the excitation is a vertical source extending from the ground plane to the base of the conducting patch. It is electromagnetically coupled with the heat sink through the substrate. There is no grounding point for this Intel P4 CPU heat sink model. Figure 4 illustrates the far field radiation pattern for the $\mathrm{E}$ field radiated from the Intel P4 CPU heat sink at $2.6 \mathrm{GHz}$.

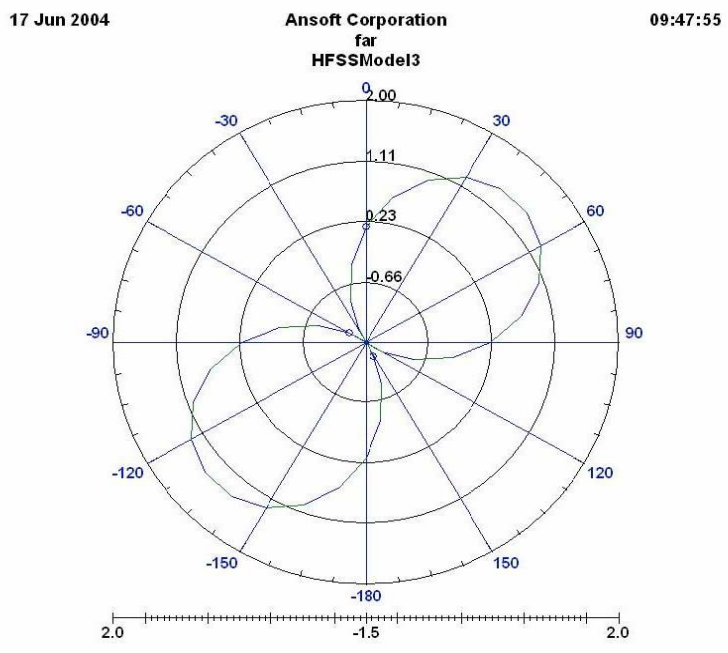

Fig. 4. Intel P4 CPU heat sink far field radiation pattern at $2.6 \mathrm{GHz}$ for the $\mathrm{E}$ theta field at 0 degree.

Figure 5 shows the swept frequency results, where two resonant frequencies were found at $2.6 \mathrm{GHz}$ and $4.5 \mathrm{GHz}$ respectively. From the reflection coefficient, $\mathrm{S}_{11}$ at $2.6 \mathrm{GHz}$ we can predict that Intel P4 CPU heat sink works as a perfect antenna and thus radiates energy into free space. A frequency sweep technique is used in the simulation considering wireless computing bandwidth. But there are some computation errors at low frequency range, such as reflection coefficient is larger than 0 . This is a typical computation problem in frequency domain when the mash size and space between

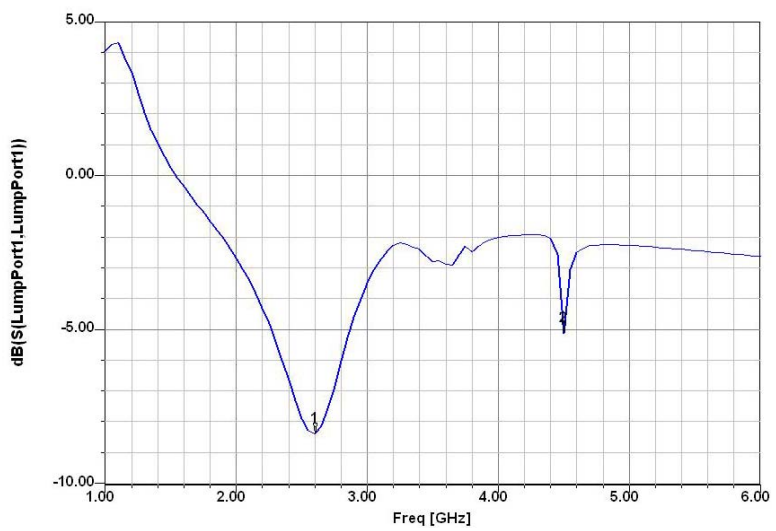

Fig. 5. Reflection coefficient, $\mathrm{S}_{11}$ for Intel P4 CPU heat sink model. 
radiation source and radiation boundary were calculated based on central frequency as mentioned in section II-B. The minimum reflection coefficient is around $-8.5 \mathrm{~dB}$ which indicates that the CPU heat sink is a radiator. One can easily observe that there is a significant amount of radiated emission from this heat sink at $2.6 \mathrm{GHz}$. Figure 6 (a) and (b) show the radiated energy flowing through the coupled patch to the heat sink and free space, and the 3D far field radiation pattern for the $\mathrm{E}$ field at $2.6 \mathrm{GHz}$ respectively. There are two radiation beams in the $\mathrm{Z}$ direction, which means the beam direction is more directional. The calculated antenna gain is larger than $>8 \mathrm{dBi}$, which is higher than most of the antennas for portable mobile devices in wireless communication systems.

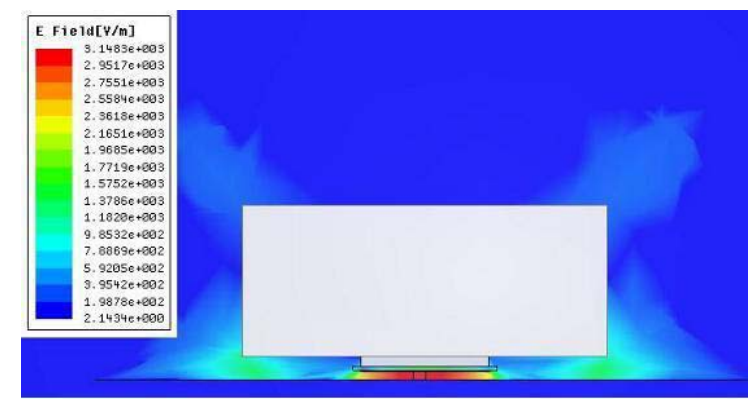

(a) Side view of the radiation energy flow observed from Intel P4 CPU heat sink with finite ground plane, where $\mathrm{E}$ field distribution is in $\mathrm{XZ}$ plane $\left(\right.$ Phase $\left.=0^{\circ}\right)$.

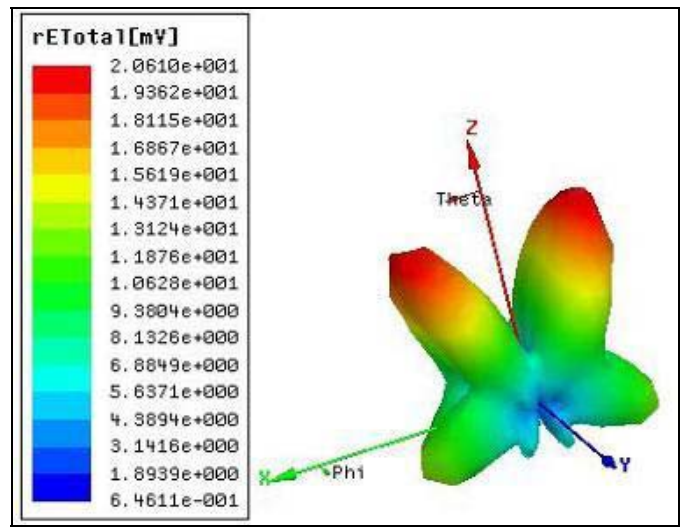

(b) Far field 3D radiation pattern

Fig. 6. 3D Simulation results of radiation pattern for E field emitted from Intel P4 CPU heat sink at $2.6 \mathrm{GHz}$.

\section{B. Analysis of IEEE EMC TC-9/2000-4 CPU Heat Sink Model}

The 2000-4 CPU heat sink problem was released in 2000 as shown in Fig. 3. Real heat sinks have fins to increase the thermal conductivity. However, the heat sink was defined as a solid block and the source was modeled as a monopole that cuts through a VLSI device. The simplified model of heat sink over a ground plane with grounding points is presented in Fig. 3 (c), where the excitation is a vertical source extending from the ground plane to the base of the heat sink, and the excitation source is slightly off center to encourage propagation of different modes. It is offset by $1.27 \mathrm{~mm}$ in the $x$ - and $y$ - directions from the center of the heat sink. The computation model is solved by FEM in the frequency domain program taking into account the absorbing boundary conditions and different grounding cases.

1) No-Grounding-Pin Case: Figures 7, 8 and 9 show the simulation results for a monopole radiator at frequency 3.15 $\mathrm{GHz}$, which is similar to the simulation results of Intel P4. The radiated emission is not that large compared with wireless communication devices. The minimum reflection coefficient is less than $-5.0 \mathrm{~dB}$ within the wireless network frequency range from $2.4 \mathrm{GHz}$ to $5 \mathrm{GHz}$.

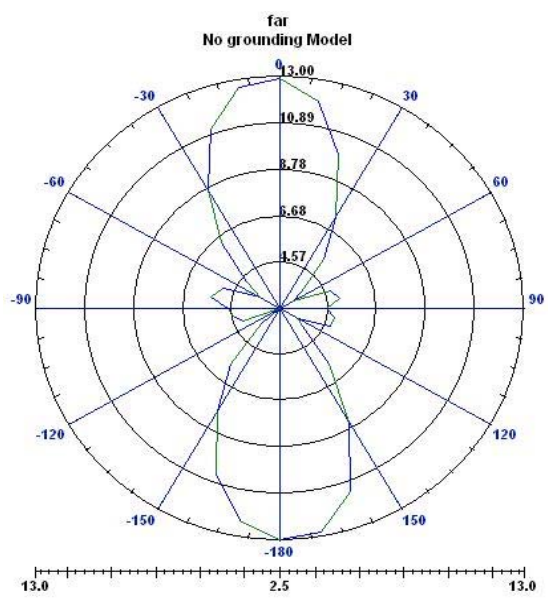

Fig. 7. Far field radiation pattern of the $2000-4$ CPU heat sink at $3.15 \mathrm{GHz}$ for the E theta field.

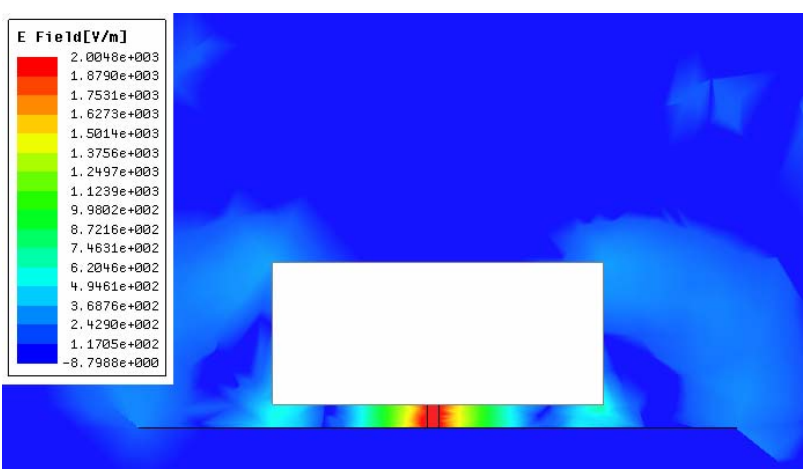

Fig. 8. Side view of the radiation energy flow observed from EMC TC9/2000-4 CPU heat sink using finite ground plane in $\mathrm{XZ}$ plane $\left(\mathrm{Phase}=0^{\circ}\right)$.

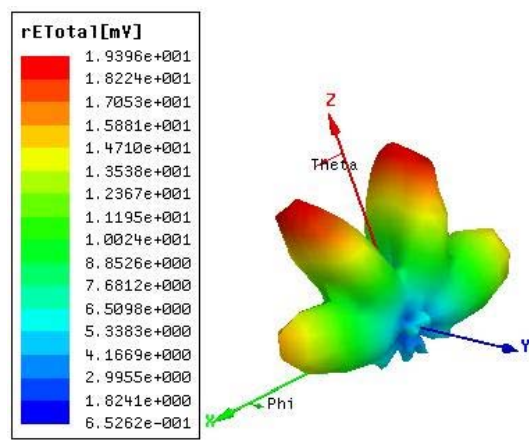

Fig. 9. 3D Simulation results of radiation pattern for $\mathrm{E}$ field emitted from EMC TC-9/2000-4 CPU heat sink at $3.15 \mathrm{GHz}$ for no-grounding-pin case. 
2) Two-Grounding-Pin Case (grounding position 1 and 2): Figures 10 and 11 show the far field radiation pattern and the reflection coefficient, $\mathrm{S}_{11}$ at frequency $3.2 \mathrm{GHz}$ respectively. Multiple resonant frequencies were found in Fig. 11 where the minimum reflection coefficient is less than $-5.5 \mathrm{~dB}$ within frequency range from $3.2 \mathrm{GHz}$ to $5.5 \mathrm{GHz}$.

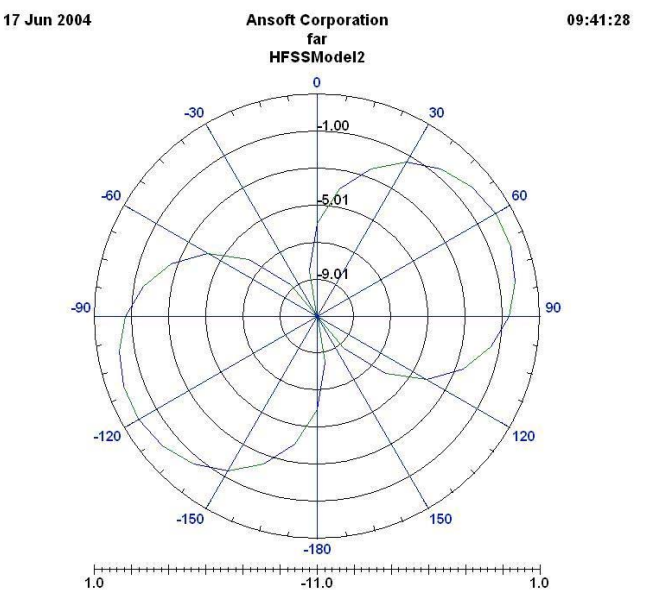

Fig. 10. Far field radiation pattern for two grounded (grounding 1 \&2) EMC TC-9/2000-4 CPU heat sink at frequency $3.2 \mathrm{GHz}$ for $\mathrm{E}$ theta field at 0 degree.

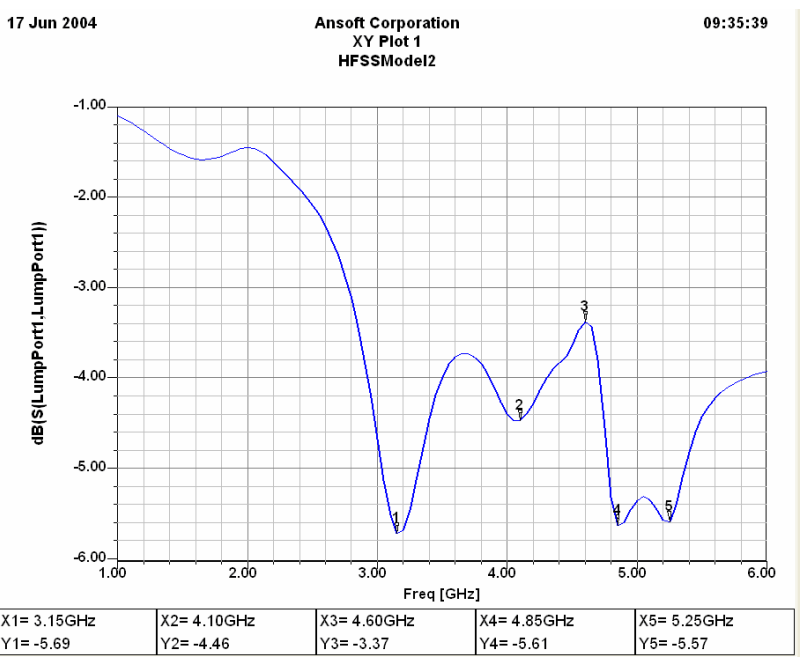

Fig. 11. Reflection coefficient, $\mathrm{S}_{11}$ for two grounded EMC TC-9/2000-4 CPU heat sink.

3) Four-Grounding-Pin Case: With four corner grounded heat sink, different results can be obtained. Figure 12 through 14 show simulation results of far field radiation pattern at $1.83 \mathrm{GHz}$, reflection coefficient and $3 \mathrm{D}$ radiation pattern related to radiated emissions from heat sink. From Fig. 13 we found that there were multiple resonant frequencies between $1.8 \mathrm{GHz}$ and $6 \mathrm{GHz}$ and the minimum reflection coefficient is less than $-13 \mathrm{~dB}$ at $5.5 \mathrm{GHz}$. Figure 14 shows that the energy was emitted from four corners.

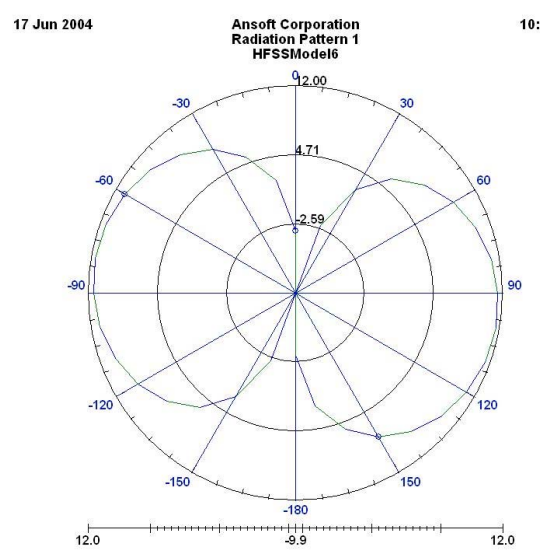

Fig. 12. Far field radiation pattern for four corner grounded EMC TC-9/2000$4 \mathrm{CPU}$ heat sink at frequency $1.83 \mathrm{GHz}$ for $\mathrm{E}$ theta field at 0 degree.

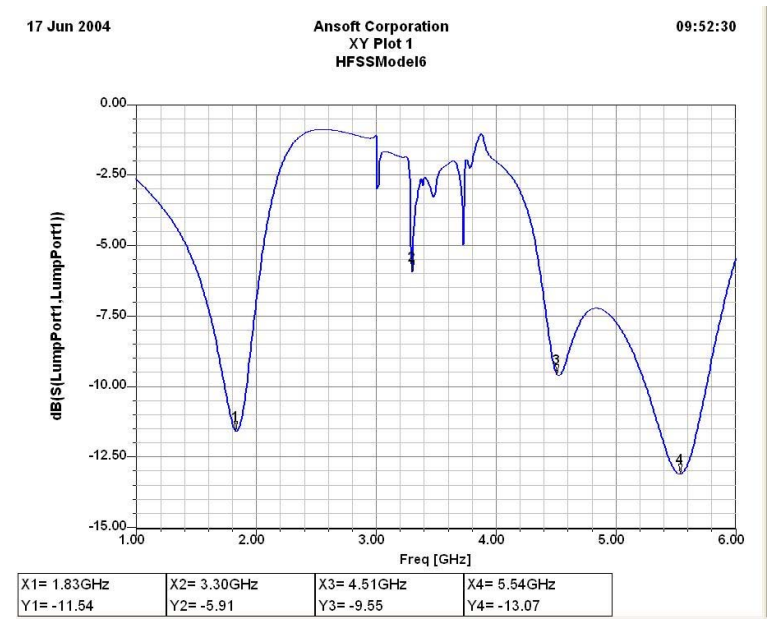

Fig. 13. Reflection coefficient, $\mathrm{S}_{11}$ for four corner grounded EMC TC9/2000-4 CPU heat sink.

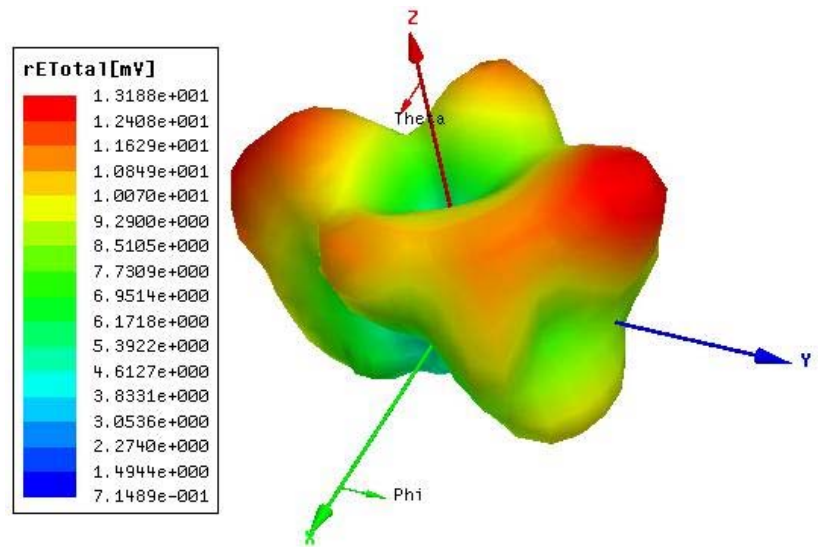

Fig. 14. 3D simulation results of radiation pattern for $\mathrm{E}$ field emitted from 2000-4 CPU CPU heat sink for four corner grounding pin case at $1.83 \mathrm{GHz}$. 
4) Comparison of frequency sweeping results: Table 1 illustrates the case studies of grounding configurations for Intel P4 CPU and IEEE EMC TC-9, 2000-4 CPU heat sinks. As illustrated in Fig. 3, several grounding positions and combinations are considered in the simulation, where the heat sink for Case I was no grounding applied to this model. Case II through Case V have used different grounding positions.

TABLE I

CASe Study Of CPu Heat SinK Model

\begin{tabular}{|c|c|c|}
\hline Case No. & CPU heat Sink & Grounding state \\
\hline CASE I & $\begin{array}{l}\text { IEEE EMC TC- } \\
9 / 2000-4\end{array}$ & No grounding \\
\hline CASE II & $\begin{array}{l}\text { IEEE EMC TC- } \\
9 / 2000-4\end{array}$ & $\begin{array}{l}2 \text { point grounding, ends } \\
\text { (pts. } 1,2 \text { ) }\end{array}$ \\
\hline CASE III & $\begin{array}{l}\text { IEEE EMC TC- } \\
9 / 2000-4\end{array}$ & $\begin{array}{l}2 \text { point grounding, edges } \\
\text { (pts. } 3,4 \text { ) }\end{array}$ \\
\hline CASE IV & $\begin{array}{l}\text { IEEE EMC TC- } \\
9 / 2000-4\end{array}$ & $\begin{array}{l}4 \text { point grounding, corners } \\
\text { (pts. } 5,6,7,8 \text { ) }\end{array}$ \\
\hline CASE V & $\begin{array}{l}\text { IEEE EMC TC- } \\
9 / 2000-4\end{array}$ & $\begin{array}{l}4 \text { point grounding, centre } \\
\text { (pts. } 1,2,3,4)\end{array}$ \\
\hline CASE VI & INTEL P4 & Insulated grounding \\
\hline
\end{tabular}

The comparison of frequency sweeping results for both Intel P4 and IEEE EMC TC-9/200-4 CPU heat sink models is shown in Fig. 15. The reflection coefficients for case III and V are $-16 \mathrm{~dB}$ at $3.6 \mathrm{GHz}$ and $-20 \mathrm{~dB}$ at $3.4 \mathrm{GHz}$ respectively. Several resonate frequencies are predicated in case IV, $\mathrm{V}$ and VI at $1.8 \mathrm{GHz}$ and $4.5 \mathrm{GHz}, 4.1 \mathrm{GHz}$, and $2.6 \mathrm{GHz}$. Obliviously the CPU heat sink can perform as an efficient radiator at these frequencies. As we mentioned in previous sections, a frequency sweep technique may cause computation errors which resulted in reflection coefficient, $S_{11}$ large than 0 at low frequency range as illustrated in Fig. 15, case II and VI. To avoid such computation error, a frequency sweep technique should be carried out within a narrow frequency range and the space between radiation source and boundary in simulation domain should use the $1 / 4$ wavelength of lowest frequency band.

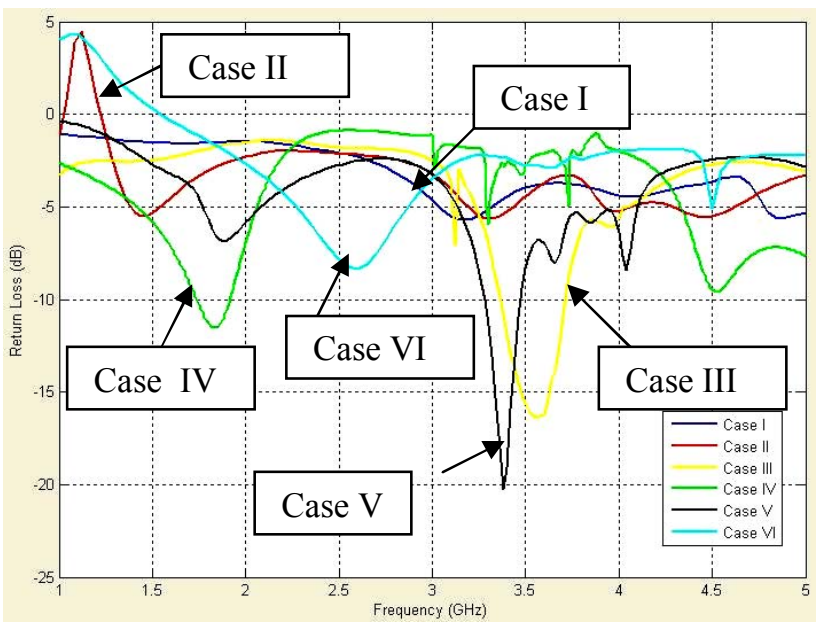

Fig. 15. Comparison of frequency sweeping results for Intel P4 CPU and IEEE EMC TC-9, 2000-4 CPU heat sink models.

\section{CONCLUSIONS}

This paper presents a finite element frequency domain method to model the radiated emissions from a CPU heat sink. Comparing the results with the EMC Challenge model or the traditional model using a grounded heat sink configuration, the P4 CPU heat sink with an insulated configuration was found to radiate emission at $2.6 \mathrm{GHz}$. The second resonant frequency was found to be at $4.5 \mathrm{GHz}$. These resonant frequencies, within the wireless computing frequency range, are associated with the heat sink structure and the CPU clock speed. Future work is to reduce the radiated emission from the heat sink by optimizing the physical size of the heat sink and insulation structure. An optimal design of the CPU heat sink should be performed in future in order to minimize the radiated emission from the CPU heat sink.

\section{REFERENCES}

[1] K. Li and et al, "Application of FD-TD Method to Analysis of EM Radiation from VLSI Configurations," IEEE Trans. On EMC, vol. 35, No. 2, pp 204-213, May 1993.

[2] B. Archambeault, C. Brench and O. M. Ramahi, EMI/EMC Computational Modeling Hand Book, Second Edition, Kluwer Academic Publishers, 2001.

[3] P.Zhou, J.Lu and et al, EMC Computer Modeling, China Electric Power Industry Publisher, Electric Power Industry Press, Feb. 2006.

[4] IEEE/EMC TC-9 and ACEM, http://aces.ee.olemiss.edu/

[5] C.E.Brench, "Heatsink Radiation as a Function of Geometry," IEEE Symposium on EMC, pp. 105-109, 1994.

[6] S.K. Das and T.Roy, "An Investigation of Radiated Emissions from Heatsinks, IEEE Symposium on EMC,” vol. 2 pp. 784-789, 1998.

[7] Intel Corporation, Intel Pentium 4 Processor in the 478-Pin Package, pp47, April 2002

[8] J.Lu and D. Xiao, "EMC Computer Modeling for CPU Heat Sink Simulation," Proceedings of ICCEA2004, pp.272-275, Dec. 2004.

[9] J.Lu and F. Dawson: "EMC Computer Modeling Techniques for CPU Heat Sink Simulation," IEEE Trans. On MAGNETICS, vol. 42, No. 10, pp 3171-3173, OCT. 2006.

[10] HFSS v9.0. (2004). Ansoft Corporation: http://www.ansoft.com/products/hf/hfss/ 